\title{
Risk of fall (RoF) intervention by affecting visual egocenter through gait analysis and yoked prisms
}

\author{
William V. Padula ${ }^{\mathrm{a}, *}$, Prem Subramanian ${ }^{\mathrm{b}}$, April Spurling ${ }^{\mathrm{a}}$, William V. Padula ${ }^{\mathrm{c}}$ and Jonathan Jenness ${ }^{\mathrm{a}}$ \\ ${ }^{a}$ Padula Institute of Vision Rehabilitation, Guilford, CT, USA \\ ${ }^{\mathrm{b}}$ Department of Ophthalmology, University of Colorado School of Medicine, Aurora, CO, USA \\ ${ }^{\mathrm{c}}$ Department of Health Policy and Management, Johns Hopkins Bloomberg School of Public Health, \\ Baltimore, MD, USA
}

\begin{abstract}
.
BACKGROUND: Following a neurologic event such as traumatic brain injury (TBI), cerebrovascular accident (CVA), and chronic neurological conditions including Parkinson's disease, multiple sclerosis, and cerebral palsy a shift in the visual midline (egocenter) can directly affect posture, balance and spatial orientation. As a consequence, this increases the risk of fall (RoF) and injury that imposes a major financial burden on the public health system.

OBJECTIVE: To determine if there is a statistically significant change in balance with the intervention of yoked prisms to reduce the risk of fall in subjects with neurological impairments.

METHODS: Ambulation of thirty-six subjects was evaluated on a pressure sensitive mat before and after intervention with yoked prisms. Changes in gait and balance were analyzed in the anterior-posterior (AP) and medial-lateral (ML) axes during ambulation. RESULTS: $T$-tests for each measure comparing the difference-of-differences to a zero change at baseline returned statistically significant reductions in both $\mathrm{AP}(p<0.0001 ; 95 \%$ CI: 1.368-2.976) and ML ( $p=0.0002 ; 95 \%$ CI: $1.472-4.173)$ imbalances using specifically directed yoked prisms to correct the visual midline deviation.

CONCLUSION: These findings demonstrate that yoked prisms have the potential to provide a cost-effective means to restore the visual midline thereby improving balance, reduce RoF and subsequent injury.
\end{abstract}

Keywords: Visual midline shift syndrome, yoked prisms, balance, spatial visual process, visual egocenter

\section{Introduction}

Falls are a major public health risk (Centers for Disease Control, 2004). Each year over 37 million people seek medical attention for a fall related injury costing 19 billion dollars for injuries sustained in the year 2000 (Stevens, Corso, Finkelstein, \& Miller, 2006). In the U.S., 25\% of all hospital admissions are for injuries secondary to falls (Centers for Disease Control, 2004).

\footnotetext{
*Address for correspondence: William V. Padula, Padula Institute of Vision Rehabilitation, 37 Soundview Rd., Guilford, CT, USA. Tel.: +1 203453 2222; Fax: +1 203458 3463; E-mail: wpadula@padulainstitute.com.
}

One in three people over age 65 will sustain a fall each year (Trump, Pluijm, \& Smit, 2001). Considering older adults, falls are the most common cause of injuries and hospital admissions for trauma which accounts for $87 \%$ of all fractures in patients who are 65 years and older (Fife \& Barancik, 1985). Added to this concern is that $53 \%$ of those adults discharged from medical care for fall-related hip fractures will experience another fall within 6 months, developing increased risk for chronic morbidity according to Creditor (Creditor, 1993).

Neurologic events such as traumatic brain injuries (TBI), cerebrovascular accidents (CVA), and chronic neurological conditions including Parkinson's disease, 
multiple sclerosis, and cerebral palsy can directly affect posture, balance and spatial orientation; thus, these conditions significantly increase patients' risk of fall (RoF) (National Hospital Ambulatory Medical Survey, 2015). The prevalence of TBI and CVA is increasing rapidly in the United States, with an estimated 50 million people suffering from CVA, and an additional 10 million from TBI (Padula, Argyris, \& Ray, 1994).

According to Padula et al., visual midline (egocenter) is normally established during early development of the central nervous system and allows proper alignment of the body's center of mass (CM) to maintain balance during mobility (Padula, Nelson, Benabib, Padula, Yilmaz, \& Krevisky, 2009). The spatial visual process interacts with the proprioceptive base of support (BOS) and the vestibular process to organize postural alignment upright against gravity. The visual midline (VM) is a result of this match of spatial information.

A mismatch between the visual spatial information and the proprioceptive BOS following a neurological event such as a CVA or TBI can compromise the relationship of spatial orientation upright against gravity, thereby causing a shift in visual midline and affecting the center of mass (CM) in the human body (Padula, et al., 2009). The midline perceptual deficit, called the Visual Midline Shift Syndrome (VMSS), may not be detected by the standard clinical examination. Thus, patients undergoing physical or occupational therapy after an acute event, such as a CVA or TBI, may fail to make the expected progress due to the disruption of the spatial visual process, and causing VMSS. They will often be discharged because of their lack of progress.

In addition to CVA or TBI, the aging process itself may alter the perception of the midline. A shift in the VM affects the position of the CM during ambulation. This abnormal condition causes difficulty with weight shift during ambulation and affects balance and posture. Depending on the severity of the VMSS, RoF and subsequent injury is increased.

Padula et al. (2009) demonstrated that the spatial visual process supports balance and stabilization of the $\mathrm{CM}$ by establishing a match of the VM in relationship to the proprioceptive BOS (Padula et al., 2009). The study further proved that $\mathrm{CM}$ is affected by the use of yoked prisms that normalize the position of the VM. This enhances proper weight shift and balance when standing or during ambulation.

Weight shift affects the center of gravity (CG). The $\mathrm{CG}$ is the average location of the weight of an object. The CM is the point from which all of the body's mass is equally distributed. An alteration of the CG and CM interferes with the balance between the spatial visual process and the base of support (BOS), in turn, affecting RoF. The CM is located approximately 2 inches below the navel and is supported by the BOS of the two legs. During dynamic movement occurring ambulation, the CM relationship to the BOS is critical in maintaining balance as the $\mathrm{CG}$ shifts with weight transfer to the supporting foot and leg. When the CM becomes displaced with an abnormal weight shift, there is a lack of counter balance needed for the transfer of the CG from single support (standing on one foot) to the weight distribution required for transferring weight from the toe of one foot to the heel strike of the other foot in double support.

A prism is a wedge of optical media incorporating a base and apex that compresses space in one direction and expands space in another. Prisms are traditionally used to compensate for a deviation in eye alignment. However, yoked prisms as used in this study are not intended for the typical compensation, but are rather purposed to affect one's position sense and orientation to body space as documented by Eubank (Eubank \& Ool, 2001). Yoked prisms expand and compress space equally for both eyes affecting VM and, in turn, the CM. For patients impaired by the above neurological events, the benefit of yoked prisms becomes an important rehabilitative tool in neutralizing the sensorimotor distortion caused by the mismatch of the affected (paretic) and non-affected sides. Research has documented the benefit of yoked prisms improving spatial neglect and spatial orientation (Milner, Dijkerman, McIntosh, Rossetti, \& Pisella, 2003; Rode, Pisella, Marsal, Mercier, Rossetti, \& Boisson, 2006; Tilikete, Rode, Rossetti, Li, Pichon, $\&$ Boisson, 2001).

The intent of the present study is to demonstrate that improvement in weight shift and stabilization of the $\mathrm{CM}$ by manipulating the VM through yoked prisms intervention is an effective means to improve balance. It is hypothesized that yoked prisms will shift the perceived $\mathrm{VM}$ in the anterior/posterior (AP) and/or medial/lateral (ML) axes to realign the CM thereby improving balance and potentially reducing RoF.

\section{Methods}

\subsection{Study design}

A clinical intervention study was performed on thirty-six subjects. The subjects were evaluated while ambulating on a customized pressure-sensitive mat 
(ProtoKinetics Zeno Walkway, ProtoKinetics LLC, Havertown, PA, (02015) before and after introduction of yoked prisms to measure changes in gait and balance associated with VMSS. All subjects initially reported difficulty with balance as a result of their diagnosed neurological condition. Each subject's gait and balance were measured during three passes on the mat. Table 1 shows the various diagnosed conditions affecting balance with these subjects.

\subsection{Inclusion criteria}

Subjects diagnosed with CVA (ICD-10 I63.X), concussion (ICD-10 F07.81), multiple sclerosis (ICD-10 G35), vertigo (ICD-10 R42), Lyme disease (ICD-10 A69.22), essential tremor (ICD-10 G25.0), brain tumor (ICD-10 D33.2) and who were between the ages of 18-80 were included in this study. The diagnosed conditions of each subject manifested a variety of postural/balance related abnormalities. All subjects reported difficulty with balance and were subject to falls. Subjects were selected after appearing to have possible Visual Midline Shift Syndrome (VMSS) causing drifting or abnormal weight shift while ambulating. Subjects were excluded if they were not able to ambulate on the gait mat independently.

\subsection{Gait analysis}

The pressure sensitive mat has an active area of $2 \mathrm{ft} . \times 16 \mathrm{ft}$. and 18,432 pressure sensitive cells (see Figs. 1 and 2). As the subject ambulates across the mat, the force exerted by the feet activates the internal pressure sensitive cells. The information is recorded in pre-determined time intervals by the electronics on one side of the outer perimeter of the walkway mat. For every activated sensing cell the hardware delivers

Table 1

Diagnosed conditions affecting balance of subjects in the study

\begin{tabular}{llr}
\hline & Method \\
\hline Number of Subjects & $36(13$ male-23 female $)$ & 3 \\
\hline Diagnosed Condition & Chiara Malformation & 8 \\
& Lyme disease & 10 \\
& Concussions & 2 \\
& Multiple Sclerosis & 9 \\
& CVA & 1 \\
& Brain tumor & 1 \\
& Essential tremor & 1 \\
& Vertigo & 1 \\
Average Age & Undiagnosed Etiology & \\
Female (aver. Age) & 39 yr. & \\
Male (aver. Age) & 36 yr. \\
\hline
\end{tabular}

the coordinates ( $\mathrm{X}$ and $\mathrm{Y}$ ), time of activation, and the relative pressure level exerted on the activated cell.

Data were analyzed using ProtoKinetics Movement Analysis Software (ProKinetics LLC, Havertown, PA, (C)2015). The data collected for this analysis included 104 variables, with the cyclogram parameter being used as the primary outcome measure. A cyclogram is a graphical representations of the data recorded from pressure exerted in the anterior/posterior (AP) and medial/lateral (ML) directions (see Fig. 3). These measurements were chosen for this study because they represent the dynamics of smooth gait weight transfers, symmetry of gait, and sensitivity to changes with any intervention.

\subsection{Testing methods}

Subjects wore their habitual spectacle correction for the pre-intervention analysis while ambulating on the pressure sensitive mat for three passes. Following this trial, subject data from the pressure sensitive mat were corrected for missteps manually. The data was then analyzed for skew in AP and ML weight shift. The unit scale for the AP and the ML axes was normalized to a percentage value. Each footprint recorded by the pressure sensitive cells used the largest length and width value for that foot. This information was averaged thereby establishing a Center of Origin or reference point between the left and right footfalls. This was then used to evaluate differences in the shift of weight (Fig. 3).

The AP and ML unit value percentage is a representation in the deviation of the intersection point between the transfer of weight from single support to double support. The intersection point where double support

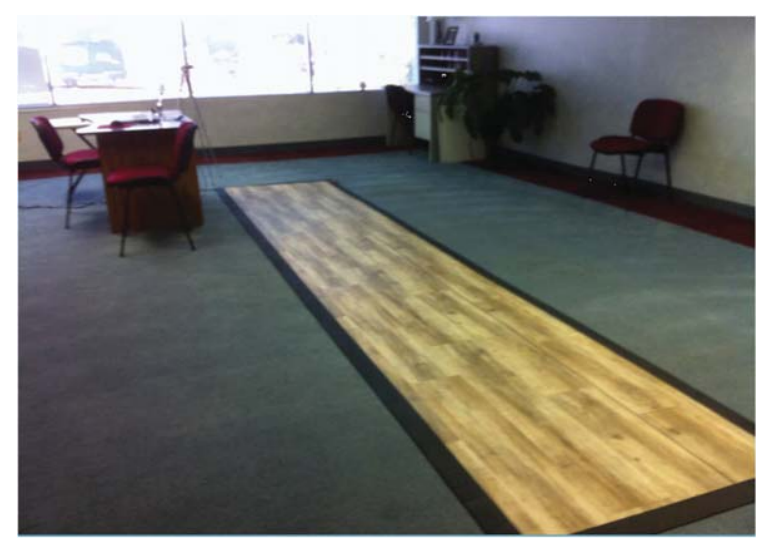

Fig. 1. Computerized walkway mat. 


\section{Baseline Walking}

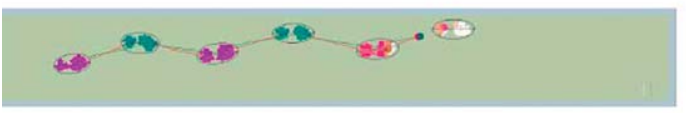

\section{Center of Mass estimated Velocity}

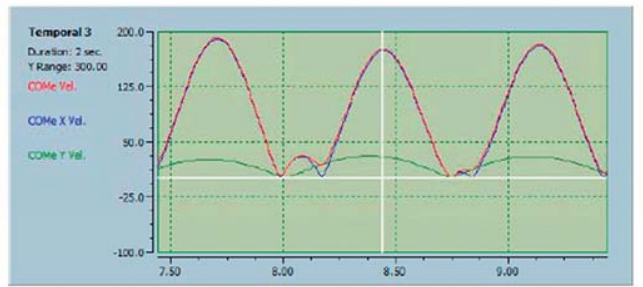

Center of Mass estimated Acceleration

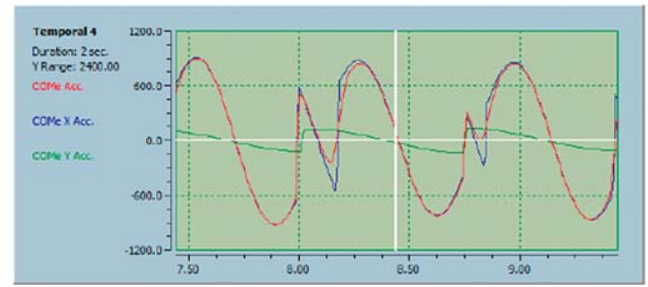

Prism Glasses

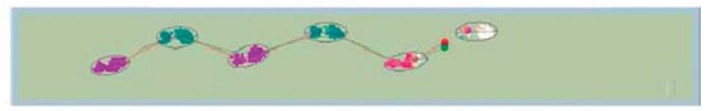

Center of Mass estimated Velocity

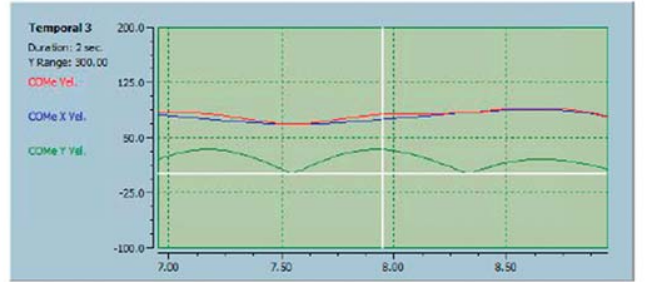

Center of Mass estimated Acceleration

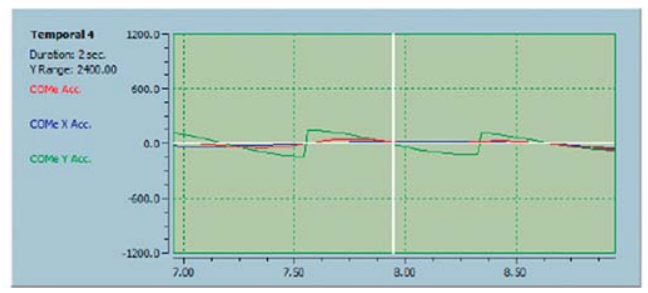

Fig. 2. This figure demonstrates that the variability of the center of Mass (COM) estimated velocity and acceleration reduced significantly by introducing the yoked prism lenses.

is established during transition from the right toe to left heel and the left toe to the right heel represents the terminal point of double support and is termed the CISP-AP (anterior-posterior) and CISP-ML (mediallateral). The CISP-AP and the CISP-ML describe the shift of the Center of Pressure (COP) from the Center of Origin Deviation in the anterior-posterior axis is designated with a negative sign (-) if the CISP-AP is shifted posteriorly and positive (+) for an anterior shift. If the CISP-ML is shifted to the right it is designated with a negative sign (-) and if it is deviated to the left a positive (+) value is applied.

Each subject was then fitted with a pair of yoked prism lenses inserted into a trial frame in conjunction with the corrective lenses. The subjects repeated the walk on the mat for three passes. The prisms were oriented with the base opposite the direction of weight shift representative of the CISP-AP and CISP-ML deviation to compensate for the imbalance thereby improving gait. The changes in CISP-AP and CISP-ML were recorded.

The ratio of the imbalance of the CISP-AP and CISPML from the Center of Origin was used to calculate the power and axis of the yoked prisms used in the trial.
Figure 4 is a graphical representation of the algorithm for the ratio used to calculate the axis of the yoked prisms and the dioptric amount of the prisms used for each subject. The $\mathrm{x}$-axis represents the positive (+) and negative (-) CISP-ML shift. The y-axis shows the positive (+) and negative (-) shift in the CISP-AP. The CISP-AP and ML were adjusted for the relative value of the deviation from the Center of Origin to yield the axis of the yoked prisms placed in a trial frame for each subject and the dioptric power was represented by the value of the ratio between the AP and ML.

The shift of image by the prisms is not relevant to the effect on posture and balance. Yoked prisms as described in this study affect the orientation of the spatial visual process to the proprioceptive BOS by realigning the VM relative to the BOS.

\subsection{Data analysis}

CISP-AP and CISP-ML were measured for each walking trial on the mat. The mean and standard deviation were calculated for the pre- and post-intervention recordings, and the difference was determined. Statistical significance was determined using a two-tailed $t$-test with $p \leq 0.05$ considered as significant. 


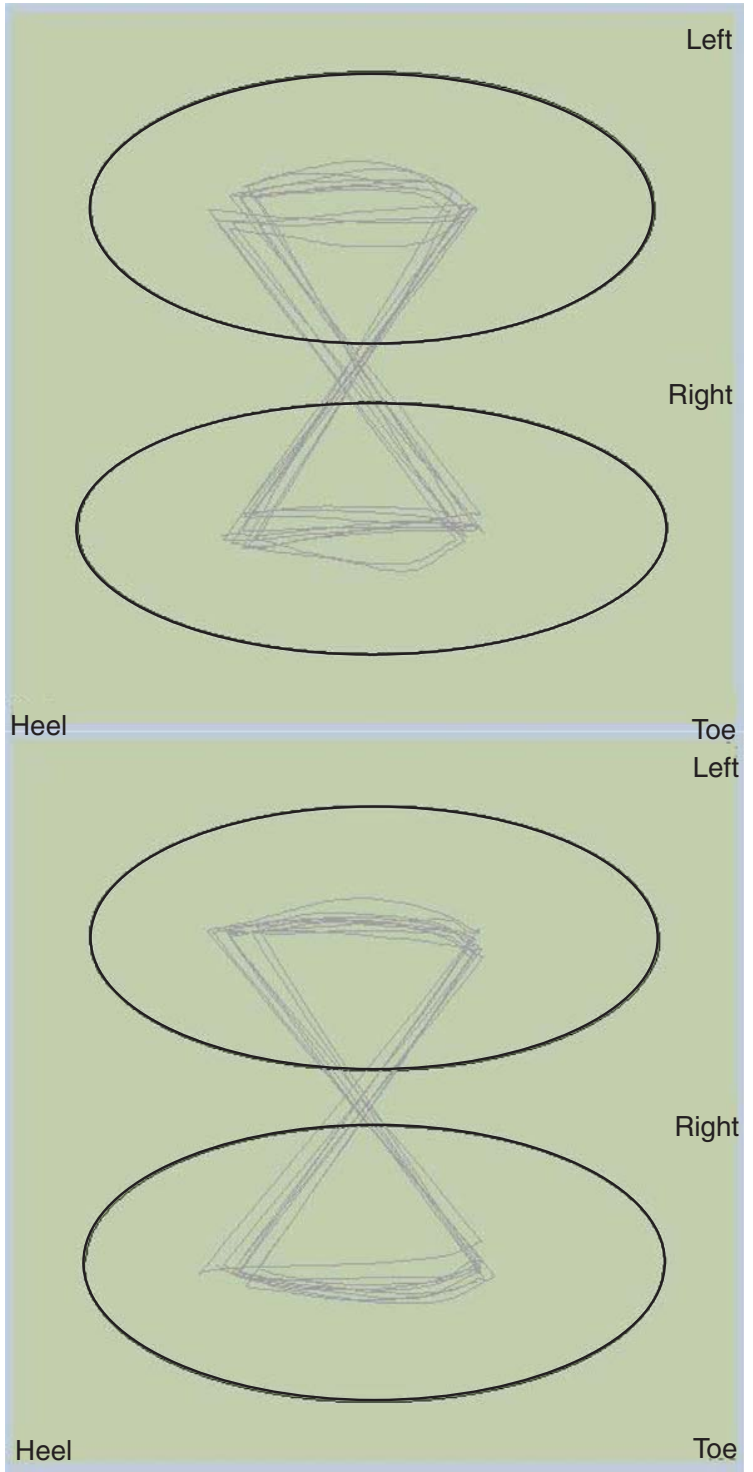

Fig. 3. This figure presents a before (top) and after (bottom) intervention with yoked prisms Cyclogram demonstrating change in the CISP-AP and ML.

\section{Results}

\subsection{Descriptive statistics}

The trial measured changes in gait and balance for 36 subjects (13 male, 23 female) with reported neurological dysfunctional symptoms from various diseases (Table 1). The average age for all subjects was $39 \mathrm{yrs.}$ with females having an average age of $36 \mathrm{yrs}$. and males having an average age of $44 \mathrm{yrs}$.

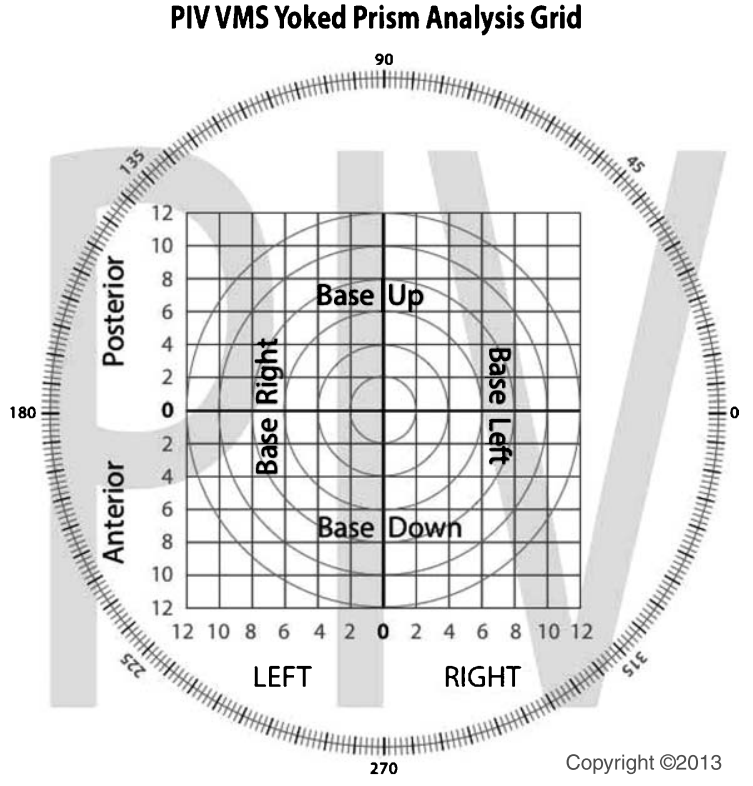

Fig. 4. The graphical representation of the algorithm used for determination of the degree axis and power of yoked prisms.

Table 2 demonstrates the results for Trial 1 and Trial 2. Trial 1 shows the deviation in the CISP-AP and CISPML with subjects without yoked prisms. Trial 2 shows the results of the change in CISP-AP and CISP-ML following intervention with yoked prisms.

Table 3 exhibits the difference-of-differences between the data from Trial 1 and Trial 2. The reason that this statistical analysis was chosen was that the CISP-AP and ML ranged from 0 to 40 . A change from 40 to 20 compared from 4 to 2 would not be recognized as a 2 to 1 improvement for each without using the difference-of-differences analysis.

Table 4 shows the statistical effect of intervention with yoked prisms. Based on a difference-of-difference analysis, the mean change in AP following intervention with yoked prisms is $2.172(\mathrm{SD}=2.375)$, and for ML the mean change is $2.823(\mathrm{SD}=3.99)$. There was slightly greater variance in the ML group than AP group. $T$-tests for each measure comparing the difference-of-differences to a zero change at baseline returned statistically significant reductions in both AP $(p<0.0001$; 95\% CI: $1.368-2.976)$ and $\operatorname{ML}(p=0.0002$; 95\% CI: $1.472-4.173)$.

Figure 3 shows the before and after representation of the shift of CISP-AP and ML following introduction of yoked prisms to stabilize and center the visual midline thereby affecting CM. The shift of the CISP-AP and 
Table 2

Data collected for the CISP AP and ML for Trial 1 and 2 in the Experimental and Control Group without yoked prisms

\begin{tabular}{|c|c|c|c|c|}
\hline \multirow[t]{2}{*}{ Subject } & \multicolumn{2}{|c|}{ Anterior/Posterior (AP) } & \multicolumn{2}{|c|}{ Medial/Lateral (ML) } \\
\hline & Trial 1 (Control) & Trial 2 (Exper.) & Trial 1 (Control) & Trial 2 (Exper.) \\
\hline 1 & -16.834 & -13.559 & 2.298 & 0.134 \\
\hline 2 & -5.827 & 0.083 & 2.917 & 1.66 \\
\hline 3 & -1.059 & 0.488 & 0.94 & 0.053 \\
\hline 4 & 7.762 & 0.515 & 1.657 & 1.001 \\
\hline 5 & 5.564 & 3.579 & 3.761 & 1.914 \\
\hline 6 & -11.456 & -10.584 & 0.528 & 0.912 \\
\hline 7 & 3.516 & 1.974 & 0.927 & 6.344 \\
\hline 8 & 3.258 & 2.293 & 5.439 & 1.714 \\
\hline 9 & 7.762 & 0.515 & 1.657 & 1.0 \\
\hline 10 & 9.704 & 9.631 & 10.21 & 0.202 \\
\hline 11 & 3.77 & 0.963 & 2.171 & 1.131 \\
\hline 12 & -3.304 & -0.915 & 0.373 & 5.085 \\
\hline 13 & -7.269 & -5.357 & 2.768 & 2.35 \\
\hline 14 & 5.811 & 2.658 & 4.69 & 3.277 \\
\hline 15 & 3.811 & 3.295 & 1.838 & 1.062 \\
\hline 16 & -3.642 & 1.386 & 2.189 & 1.698 \\
\hline 17 & 2.534 & 0.588 & 7.752 & 4.402 \\
\hline 18 & -7.427 & -1.278 & 4.282 & 4.636 \\
\hline 19 & 1.029 & -1.287 & -2.249 & 0.896 \\
\hline 20 & 6.801 & 2.272 & 2.151 & 3.991 \\
\hline 21 & 1.943 & 1.628 & 6.274 & 2.288 \\
\hline 22 & 7.001 & 6.782 & 8.111 & 0.824 \\
\hline 23 & 1.644 & -4.566 & 11.803 & 1.454 \\
\hline 24 & -21.862 & -14.471 & 83.792 & 64.765 \\
\hline 25 & -1.045 & -2.634 & 83.66 & 81.192 \\
\hline 26 & -5.309 & -1.969 & 4.808 & 1.711 \\
\hline 27 & -1.959 & -1.511 & 8.881 & 1.715 \\
\hline 28 & -11.456 & -10.584 & 0.528 & 0.912 \\
\hline 29 & 3.516 & 1.974 & 3.631 & 6.344 \\
\hline 30 & 3.144 & 0.488 & 3.696 & 0.053 \\
\hline 31 & 2.201 & 2.111 & 2.717 & 1.886 \\
\hline 32 & 10.002 & 8.771 & 4.216 & 2.313 \\
\hline 33 & 2.039 & 1.784 & 3.553 & 2.804 \\
\hline 34 & -15.936 & -13.559 & 5.136 & 2.804 \\
\hline 35 & -7.66 & -4.618 & 2.809 & 0.134 \\
\hline 36 & 1.22 & -5.136 & 2.8 & 0.553 \\
\hline
\end{tabular}

ML is a graphical representation of the corresponding data points of one subject shown in Table 2.

\section{Discussion}

According to Trevarthen, evidence of vision as a bimodal processing system composed of both focal and ambient vision, can be used as a component of the physiological rationale for why people have increased risk of fall (Trevarthen, 1968). The focal visual process is largely concerned with detail discrimination and isolates on points or sectors. The process of focalization is referenced primarily to occipital cortical function. The ambient process supports the focal process by organizing the spatial domain for posture and balance (e.g. utilizing the framework of the visuo-spatial environment to match with sensorimotor information for body-space orientation and postural alignment upright against gravity).

Compromise to the ambient process affects feed forward and spatial mapping (Benabib \& Nelson, 1993). It is analogous to seeing the 'trees' but not the 'forest', or as some persons with traumatic brain injury (TBI) describe, they can see what they attend to in the room but not the room. Up to $20 \%$ of visual information may be directed to the ambient system, particularly the midbrain where visual information is matched with kinesthetic, proprioceptive and vestibular sensorimotor information (Moore, 1993). The superior colliculus (SC) receives nerve fibers from the optic tract through the superior brachium, from the occipital 
Table 3

The table demonstrated the difference of differences between data from Trial 1 and 2

\begin{tabular}{|c|c|c|c|c|}
\hline \multirow[t]{2}{*}{ Subject } & \multicolumn{2}{|c|}{ Anterior/Posterior (AP) } & \multicolumn{2}{|c|}{ Medial/Lateral (ML) } \\
\hline & Trial 1 (Control) & Trial 2 (Exper.) & Trial 1 (Control) & Trial 2 (Exper.) \\
\hline 1 & 0 & 3.275 & 0 & 2.164 \\
\hline 2 & 0 & 6.657 & 0 & 1.257 \\
\hline 3 & 0 & -0.571 & 0 & 0.41 \\
\hline 4 & 0 & 7.247 & 0 & 0.657 \\
\hline 5 & 0 & 1.967 & 0 & 1.847 \\
\hline 6 & 0 & 0.872 & 0 & -0.384 \\
\hline 7 & 0 & 1.542 & 0 & 5.432 \\
\hline 8 & 0 & 0.965 & 0 & 3.725 \\
\hline 9 & 0 & 7.247 & 0 & 0.474 \\
\hline 10 & 0 & 0.073 & 0 & 10.008 \\
\hline 11 & 0 & 2.807 & 0 & 1.04 \\
\hline 12 & 0 & 2.389 & 0 & 2.164 \\
\hline 13 & 0 & 1.912 & 0 & 1.257 \\
\hline 14 & 0 & 3.153 & 0 & 0.41 \\
\hline 15 & 0 & 0.516 & 0 & 0.657 \\
\hline 16 & 0 & 2.256 & 0 & 1.847 \\
\hline 17 & 0 & 1.946 & 0 & -0.384 \\
\hline 18 & 0 & 6.149 & 0 & 5.432 \\
\hline 19 & 0 & 0.247 & 0 & 3.725 \\
\hline 20 & 0 & 4.529 & 0 & -1.84 \\
\hline 21 & 0 & 0.315 & 0 & 3.986 \\
\hline 22 & 0 & 0.218 & 0 & 7.287 \\
\hline 23 & 0 & -2.922 & 0 & 10.349 \\
\hline 24 & 0 & 7.391 & 0 & 19.027 \\
\hline 25 & 0 & 1.589 & 0 & 2.468 \\
\hline 26 & 0 & 3.348 & 0 & 3.097 \\
\hline 27 & 0 & 0.448 & 0 & 7.166 \\
\hline 28 & 0 & 0.872 & 0 & -0.384 \\
\hline 29 & 0 & 1.542 & 0 & -2.713 \\
\hline 30 & 0 & 2.656 & 0 & 3.643 \\
\hline 31 & 0 & 0.09 & 0 & 0.831 \\
\hline 32 & 0 & 1.229 & 0 & 1.903 \\
\hline 33 & 0 & 0.255 & 0 & 0.749 \\
\hline 34 & 0 & 0.564 & 0 & 2.332 \\
\hline 35 & 0 & 2.377 & 0 & 2.675 \\
\hline 36 & 0 & 3.042 & 0 & 2.247 \\
\hline
\end{tabular}

Table 4

Statistical analysis for the AP and ML Control vs. Experimental Groups

\begin{tabular}{lcc}
\hline & AP Control vs. Experimental & ML Control vs. Experimental N-75 \\
\hline$P$-Value & 3.143 & 4.263 \\
$T$ Value & 5.639 & 4.359 \\
Mean & 2.171 & 2.823 \\
SD & 2.374 & 3.994 \\
Degrees of Freedom & 35 & 35 \\
$\mathrm{P}$ & $<0.001 ; 95 \% \mathrm{CI}=1.368-2.976$ & $<0.0001 ; 95 \% \mathrm{CI}=1.472-4.173$ \\
$\mathrm{p}$ & $<0.05$ & $<0.05$ \\
\hline
\end{tabular}

cortex via the optic radiations through the lateral geniculate body and from the spinotectal tract completing connections with the sensorimotor systems from the spinal cord and medulla (Johannsen, Broez, \& Karnath, 2006). This interaction and matching of retinal and sensorimotor information provides the input for posture, balance, movement and positional orientation (Adams \& Hurwitz, 1963). The SC provides for the coordination of eye movements with movement of the body and head (Bron, Tripathi \& Tripathi 1997; Perennou, 2006). This enables awareness of our surroundings while maintaining the ability to fixate on an object. 
This balance should not be compromised even when our central awareness of the object comes from an auditory rather than a visual cue (Perennou, 2006; Rolfs \& Ohl, 2011).

Himmelbach et al. (2007) demonstrated that involvement of the superior colliculus in visual search tasks supports a dependency of superior colliculi activity on functions beyond oculomotor control and visual processing (Himmelbach, Erb, \& Karnath, 2007). A bimodal processing model enabled Milner et al. (2003) to predict that cortical dorsal streaming mediates normal visual-guided actions, while ventral streaming provides visual information that is memory-based (Miler et al., 2003). Spatial information from the ambient visual process, delivered by the superior colliculus, provides the binding format of the fusion process for integrating the images from the right and left eyes (Nashold, \& Seaber, 1972).

Dysfunction of the ambient visual process in relationship to mismatch between sensorimotor information also relates to concepts of visual midline organization (e.g. the pre-conscious ambient visual concept of the lateral and anterior-posterior axes related to the perceived body center for sense of position) (Padula et al., 1994; Zasler, Katz, \& Zafonte, 2013). Rossetti et al. determined that pathological shift of the subjective midline will occur relative to left hemispatial neglect and that treatment with yoked prisms demonstrated improvement or reduction in neglect (Rossetti et al., 1998).

The yoked prisms reliably improved the secure weight bearing of the subjects in both the AP and ML axes. This improvement supports the hypothesis that VMSS is indeed a valid nosologic entity associated with brain injury and dysfunction following a neurologic event, as suggested in previous studies. Furthermore, the results support the concept that a shift in the visual midline shift negatively reinforces abnormal weight bearing that prevents and/or interferes with optimal rehabilitation.

The etiology of VMSS is currently unknown. It has been hypothesized that a neurological event such as TBI or CVA causes abnormal processing of preconscious visuo-spatial information with the proprioceptive BOS and its interaction with the conscious organization of visual information. This leads to a mismatch between visual spatial preconscious organization and the proprioceptive BOS. In turn, the mismatch or spatial distortion produces the self-reinforcing VMSS. In VMSS, the proprioceptive inputs are abnormally coordinated with visuo-spatial input. This affects spatial mapping relative to the frontal supplementary eye fields, posteriorparietal lobe and the occipital cortex.

\section{Conclusion}

This study demonstrates the following: 1. Imbalances between weight shift for the CISP-AP and ML were reduced by intervening with yoked prisms designed with base end of the prisms positioned in the direction opposite to the direction of weight shift; 2 . The reduction in abnormal weight shift from use of yoked prisms was found to be statistically significant; 3 . Balance can be affected through application of yoked prisms that influence the visual spatial process thereby reducing RoF; and, 4. Weight shift analysis of single and double support is an effective means to analyze and calculate the corresponding value of yoked prisms to be prescribed to reduce RoF and improve balance. Therefore, yoked prisms are an effective means to influence balance and RoF for persons with a neurological condition.

This research demonstrates that by measuring the degree and direction to which patients with neurological event abnormally weight shift (using weight bearing analysis), one can estimate the deviation in VM and provide appropriate yoked prisms to alter this imbalance. The statistical significance demonstrates that yoked prisms oriented with the base end of prisms opposite the weight shift and as represented by the CISP-AP and CISP-ML are an effective means to realign the deviation of the CM.The reason for this is that the VMS is a distortion in the spatial visual process relative to the proprioceptive BOS. This causes a VM shift affecting the $\mathrm{CM}$ and an increase in weight bearing or pressure that is skewed in comparison to where the physical center is located. The shift in VM affects the CG and influences abnormal shift in weight bearing.

In this study all patients were fitted with yoked prisms oriented to alter the $\mathrm{CM}$ toward the physical midline; the direction of the prism was chosen based upon the initial Trial 1. Future studies to demonstrate efficacy of the technique and further validate its mechanism would include fitting patients with sham prisms or incorrectly oriented prisms. Additionally, normal controls may be fitted with yoked prisms to induce a predicted CM decentration appropriate for the prism power and direction employed. In this study, we did not evaluate elderly patients separately from the overall group. Since RoF is most significant for the aging adult, we suggest future 
studies be concentrated on subjects over 65 years of age to analyze the effectiveness of visual intervention to reduce $\mathrm{RoF}$ in this population.

In addition, it is recommended that a longitudinal study be aimed at following patients treated for VMSS through use of rehabilitative-yoked prisms to determine if the RoF continues to be reduced compared to the population of subjects with similar diagnoses and non-intervention. It would also be useful to evaluate qualitative, quantitative, and functional outcome measurements of rehabilitation in conjunction with physical and occupational therapy compared to non-intervention with prescribed rehabilitative-yoked prisms. Finally, a cost-effectiveness analysis is recommended to evaluate and compare outcome of rehabilitation with and without prescribed rehabilitative-yoked prisms. We believe that these studies could demonstrate that rehabilitativeyoked prisms represent a cost-effective means to improve quality of life, reduce RoF and subsequent injury in populations of patients where physical and occupational therapy are not available.

The implications from this research are that yoked prisms are an effective means to reduce RoF through appropriate application of intervention when postural imbalance and abnormal weight occur from aging and/or a neurological event. This research also presents the opportunity to predict that reduced RoF is possible for individuals with hemi-paresis following a CVA or TBI.

The authors further emphasize, that based on this research, use of prescribed rehabilitative and therapeutic yoked prisms will maximize the potential outcome of physical and occupational therapy for patients having balance difficulties as a result of aging and/or a neurological event.

In conclusion, this study demonstrates that a visualspatial processing dysfunction following a neurological event increases RoF. Furthermore, intervention with yoked prisms through the visual process is an effective means of improving balance and reducing RoF. Thus, we recommend patients with $\mathrm{RoF}$ who are in rehabilitation programs or who seek medical care for difficulties with balance and posture should be evaluated for visual spatial processing dysfunction and intervention with yoked prisms.

\section{References}

Adams, G., \& Hurwitz, L. J. (1963). Mental barriers to recovery from stroke. The Lancet, 282(7307), 533-537.
Benabib, R., \& Nelson, C. A. (1993). Efficiency in visual skills and postural control: A dynamic interaction. Occupational Therapy Practice, 3, 57-56.

Bron, A., Tripathi, R. C., \& Tripathi, B. J. (1997). Wolff's anatomy of the eye and orbit 8th ed. London, England: Hodder Arnold.

Centers for Disease Control and Prevention, National Center for Injury Prevention and Control (2004, Sept 18). Web-based Injury Statistics Query and Reporting System (WISQARS). Retrieved from http://www.cdc.gov/injury

Creditor, M. Hazards of Hospitalized Care (1993). Annals of Internal Medicine, 118(3), 219-223.

Eubank, T., \& Ool, T. (2001). Improving guided action and perception through use of prisms. Journal of the American Optometry Association, 72(4), 217-226.

Fife, D., \& Barancik, J. I. (1985). Northeastern Ohio Trauma Study III: Incidence of fractures. Annals of Emergency Medicine, 14(3), 244-248.

Himmelbach, M., Erb, M., \& Karnath, H. O. (2007). Activation of superior colliculi in humans during visual exploration. $B M C$ Neuroscience, 8(66), 1471-2202.

Johannsen, L., Broetz, D., \& Karnath, H. O. (2006). Leg orientation as a clinical sign for pusher syndrome. BMC Neurology, 6(30), 1471-2377.

Milner, A., Dijkerman, H., McIntosh, R., Rossetti, Y., \& Pisella, L. (2003). Delayed reaching and grasping in patients with optic ataxia. In: Prablanc C, Pelisson D, Rossetti Y, eds. Progress in brain research. Amsterdam: Elsevier, 225-242.

Moore, J. (1993). Brain atlas and function systems. Bethesda, MD: The American Occupational Therapy Association.

Nashold, B., \& Seaber, J. H. (1972). Defects of ocular motility after steriotactic midbrain lesions in man. Archives of Ophthalmology, 88(3), 245-248.

National Hospital Ambulatory Medical Care Survey (2015, Jan 4). Ambulatory Health Care Data. Retrieved from http://www.cdc. gov/nchs/ahcd.htm

Padula, W. V., Argyris, S., \& Ray, J. (1994). Visual evoked potentials evaluating treatment for post-trauma visions syndrome in patients with traumatic brain injuries. Brain Injury, 8(2), 125-133.

Padula, W. V., Munitz, R., \& Magrun, M. eds. (2012). Neuro-Visual Processing: An Integrated Model of Rehabilitation. Santa Ana, CA. Optometric Extension Program Press.

Padula, W. V., Nelson, C. A., Benabib, R., Padula, W. V., Yilmaz, C., \& Krevisky, S. (2009). Modifying postural adaptation following a CVA through prismatic shift of visuo-spatial egocenter. Brain Inj, 23(6), 566-576.

Perennou, D. (2006). Postural disorders and spatial neglect: A strong association. Restorative Neurology and Neuroscience, 24(4-6), 319-334.

Rode, G., Pisella, L., Marsal, L., Mercier, S., Rossetti, Y., \& Boisson, D. (2006). Prism adaptation improves spatial dysgraphia following brain damage. Neuropsychologia, 44(12), 2487-2493.

Rolfs, M., \& Ohl, S. (2011). Visual suppression in the superior colliculus around the time of microsaccades. J Neurophysiol, 105(1), 1-3.

Rossetti, Y., Rode, G., Pisella, L., Farne, A., Li, L., Boisson, D., \& Prenin, M. T. (1998). Prism adaptation to rightward optical deviation rehabilitates left hemispatial neglect. Nature, 395(6698), 166-169.

Stevens, J. A., Corso, P. S., Finkelstein, E. A., \& Miller, T. R. (2006). The costs of fatal and nonfatal falls among older adults. Injury Prevention, 12(5), 290-295. 
Tilikete, C., Rode, G., Rossetti, Y., Li, L., Pichon, J., \& Boisson, D. (2001). Prism adaptation to rightward optical deviation improves postural balance in left hemiparetic patients. Current Biology, 11(7), 524-528.

Trevarthen, C. (1968). Two mechanisms of vision in primates. Psychol Res, 31, 299-337.
Tromp, A. M., Pluijm, S. M. F., Smit, J. H., et al. (2001). Fallrisk screening test: A prospective study on predictors for falls in community-dwelling elderly. J Clin Epidemiol, 54(8), 837-844.

Zasler, N., Katz, D. I., \& Zafonte, R.D. eds. (2013). Brain Injury Medicine: Principles and Practice. New York, NY. 